\title{
A note on commodity contingent valuation
}

\author{
Andrés García, Javier Población, and Gregorio Serna* \\ *Facultad de Ciencias Jurídicas y Sociales, Universidad de Castilla-La Mancha, \\ Cobertizo de San Pedro Mártir, s/n, Toledo 45071, Spain. \\ Tel: + 34925268800 — 5089, E-mail: Gregorio.Serna@uclm.es
}

Received (in revised form): 17th September, 2007

Andrés García is completing his PhD in Mathematics and Economics at the University of Oviedo (Spain). He holds a Master's degree in Economics and Finance from CEMFI (Madrid, Spain) and a degree in Mathematics from the University of Oviedo.

Javier Población is an economist at the Banco de España (Spanish Central Bank); he works in the risk model group. He is completing his PhD in Economics and Finance at the University of Alicante (Spain) and holds a Master's degree in Economics and Finance from CEMFI (Madrid, Spain) in addition to a degree in Physics from the Complutense University of Madrid. He previously worked for the Spanish oil company Repsol YPF in the risk-management department.

Gregorio Serna is an associate professor of Financial Economics at the University of Castilla-La Mancha, Toledo (Spain). He holds a PhD in Economics from the University Carlos III of Madrid (Spain). He has published research papers in the Journal of Banking and Finance, European Financial Management and The Quarterly Review of Economics and Finance, among others.

\section{Practical applications}

Commodity contingent valuation typically involves complex procedures, like limit steps, partial differential equations or approximations. These ad hoc solutions can only be used in the concrete problem for which they are developed. This paper tries to review and clarify stochastic calculus in the commodity contingent valuation context, simplifying formulae and deductions. This approach allows for a relatively simple pricing of all sorts of financial derivatives on commodity prices, avoiding limit steps, partial differential equations and approximations. Moreover, it has been shown how to obtain precise estimators of prices for NYMEX WTI crude oil futures contracts, in a two-factor context.

\begin{abstract}
Given the complex dynamics of commodity prices, most of the papers on the valuation of commodity contingent claims present ad hoc solutions, which are very complex and sometimes include approximations. This paper shows how well-known techniques and results in

stochastic calculus can be used to simplify formulae and deductions. Specifically, we show how to obtain more precise estimators of the parameters in the two-factor model by Schwartz than the approximations given by
\end{abstract}

Journal of Derivatives \& Hedge Funds, Vol. 13 No. 4, 2008, pp. 311-320 (C) 2008 Palgrave Macmillan Ltd $1753-9641 \$ 30.00$ the author, which tend to overestimate the parameters. These divergences are important in the valuation of commodity contingent claims.

Journal of Derivatives \& Hedge Funds (2008) 13, 311-320. doi:10.1057/palgrave.jdhf.1850079

Keywords: stochastic calculus; commodity prices; mean-reverting processes; two-factor models; volatility 


\section{INTRODUCTION}

Since the now classical Black and Scholes ${ }^{1}$ paper, Itô calculus has become a dominant approach in dealing with financial markets. Following this approach, there have been many papers assuming that commodity prices follow a geometric Brownian motion, which allows for a straightforward extension of the Black-Scholes procedure (see, eg Brennan and Schwartz, ${ }^{2}$ Paddock et al., ${ }^{3}$ among others).

As pointed out in Schwartz, ${ }^{4}$ however, the geometric Brownian motion hypothesis implies a constant rate of growth in the commodity price and a variance of future spot prices increasing monotonically with time, which is not a realistic assumption. Even more, all models with a single source of uncertainty are not very realistic since they imply a perfect correlation between futures prices for different maturities.

This is the reason why Gibson and Schwartz, ${ }^{5}$ Schwartz ${ }^{4}$ and Schwartz and Smith ${ }^{6}$ propose a two-factor model allowing for mean reversion in short-term prices and uncertainty in the equilibrium (long-term) price to which prices revert.

Schwartz ${ }^{4}$ and Cortazar and Schwartz ${ }^{7}$ have proposed different kinds of three-factor models. More recently, Cortazar and Naranjo ${ }^{8}$ extended two- and three-factor models to an arbitrary number of factors ( $\mathrm{N}$-factor model) given a fix structure.

Due to the complex dynamic of commodity prices, however, the translation of standard valuation techniques, based on the Black-Scholes framework, is not obvious. This could explain why studies on the stochastic behaviour of commodity prices present ad hoc solutions that are very complex and sometimes include approximations. This paper shows how well- known techniques and results in stochastic calculus can be used to simplify formulae and deductions. Specifically, we show how to obtain the expressions in a simpler and accurate way, together with more precise estimates of the parameters than the approximations given by the author in the two-factor model by Schwartz. ${ }^{4}$ In general, the approximations by Schwartz tend to overestimate the parameters. These divergences are important in the valuation of commodity contingent claims.

This paper is organised as follows: The next section shows how to obtain the expressions in a simpler and accurate way and more precise estimates of the parameters in the two-factor model by Schwartz. ${ }^{4}$ The subsequent section contains the empirical implications of the differences between the precise estimates given in this paper and the approximations by Schwartz. ${ }^{4}$ Finally, the last section concludes with a summary and discussion.

\section{THE PRECISE DISCRETE-TIME ESTIMATION OF THE TWO-FACTOR MODEL BY SCHWARTZ}

We will focus on the Schwartz ${ }^{4}$ two-factor model. As proposed by the author, the model can be formulated in the following way. The spot price of a commodity at time $t, S_{t}$, and its instantaneous convenience yield, $\delta_{t}$, are assumed to follow the processes:

$$
\left\{\begin{array}{l}
\mathrm{d} S_{t}=\left(\mu-\delta_{t}\right) S_{t} \mathrm{~d} t+\sigma_{1} S_{t} \mathrm{~d} z_{1} \\
\mathrm{~d} \delta_{t}=\kappa\left(\alpha-\delta_{t}\right) \mathrm{d} t+\sigma_{2} \mathrm{~d} z_{2}
\end{array}\right.
$$

As can be appreciated, the process for the commodity price allows for a stochastic convenience yield, which follows an OrnsteinUhlenbeck mean-reverting process. The convenience yield, $\delta_{t}$, can be interpreted as the benefits achieved by the owner of the physical 
commodity that are not obtained by the owner of a futures contract, which may include the ability to profit from temporary local shortages or the ability to keep a production process running.

In this model, $\mu$ represents the long-term total return on the commodity, $\kappa$ is the meanreverting coefficient, $\alpha$ is the long-term convenience yield and $\sigma_{1}$ and $\sigma_{2}$ are the volatilities of the spot price and the convenience yield, respectively. The standard Brownian motions, $\mathrm{d} z_{1}$ and $\mathrm{d} z_{2}$, are assumed to be correlated, that is $\mathrm{d} z_{1} \mathrm{~d} z_{2}=\rho \mathrm{d} t$.

For the purposes of derivative securities valuation, we need the risk-neutral or riskadjusted processes for $S_{t}$ and $\delta_{t}$. In this model, the commodity is assumed to be an asset paying a stochastic dividend yield, $\delta_{t}$. Therefore, the riskadjusted drift of the commodity price process will be $r-\delta_{t}$, where $r$ is the risk-free interest rate. Given the non-tradable nature of the convenience yield, however, it cannot be hedged and the risk-adjusted convenience yield process will have a market price of risk $(\lambda)$ linked to it.

In this model, the author assumes for simplicity that the market price of risk, $\lambda$, is

$$
\begin{aligned}
& \operatorname{Var}^{*}\left[X_{t}\right]= \\
& \qquad \begin{array}{c}
\sigma_{1} t+2 \sigma_{1} \sigma_{2} \rho\left(1-\mathrm{e}^{-k t}-k t\right) / k^{2} \\
-\sigma_{2}^{2}\left(3-4 \mathrm{e}^{-k t}+\mathrm{e}^{-2 k t}-2 k t\right) / 2 k^{3} \\
\sigma_{1} \sigma_{2} \rho\left(1-\mathrm{e}^{-k t}\right) / k \\
+\sigma_{2}^{2}\left(1-2 \mathrm{e}^{-k t}+\mathrm{e}^{-2 k t}\right) / 2 k^{2}
\end{array}
\end{aligned}
$$

constant. However, we would expect the market price of risk to be associated to certain variables such as for example the business cycle or the level of inventories. Following the original model, in this paper the market price of risk will be assumed to be constant.
Thus, the stochastic process for the two factors under the equivalent martingale measure can be written as:

$$
\left\{\begin{array}{l}
\mathrm{d} S_{t}=\left(r-\delta_{t}\right) S_{t} \mathrm{~d} t+\sigma_{1} S_{t} \mathrm{~d} z_{1}^{*} \\
\mathrm{~d} \delta_{t}=\left[\kappa\left(\alpha-\delta_{t}\right)-\lambda\right] \mathrm{d} t+\sigma_{2} \mathrm{~d} z_{2}^{*}
\end{array}\right.
$$

where $\mathrm{d} z_{1}^{*}$ and $\mathrm{d} z_{2}^{*}$ are the standard (correlated) Brownian motions under the equivalent martingale measure. The correlation between these two Brownian motions does not change, that is, $\mathrm{d} z_{1}^{*} \mathrm{~d} z_{2}^{*}=\rho \mathrm{d} t$.

Defining $Y_{t}=\ln \left(S_{t}\right)$ and applying Itô's Lemma, under the risk-neutral measure, the model can be expressed as:

$$
\left\{\begin{array}{l}
\mathrm{d} Y_{t}=\left(r-\delta_{t}-\sigma_{1}^{2} / 2\right) \mathrm{d} t+\sigma_{1} \mathrm{~d} z_{1}^{*} \\
\mathrm{~d} \delta_{t}=\left[\kappa\left(\alpha-\delta_{t}\right)-\lambda\right] \mathrm{d} t+\sigma_{2} \mathrm{~d} z_{2}^{*}
\end{array}\right.
$$

Defining the state vector as $X_{t}=\left(Y_{t}, \delta_{t}\right)^{\prime}$ and applying the results in the appendix, it is easy to see that, under the risk-neutral measure, $X_{t}$ is normally distributed with mean and variance:

$$
\begin{aligned}
E^{*}\left[X_{t}\right]= & \left(\begin{array}{c}
\left(r-\sigma_{1}^{2} / 2-\alpha\right) t+\alpha\left(1-\mathrm{e}^{-k t}\right) \lambda / k \\
\alpha\left(1-\mathrm{e}^{-k t}\right)
\end{array}\right) \\
& +\left(\begin{array}{cc}
1 & -\left(1-\mathrm{e}^{-k t}\right) / k \\
0 & \mathrm{e}^{-k t}
\end{array}\right) X_{0}
\end{aligned}
$$

$$
\left.\begin{array}{c}
\sigma_{1} \sigma_{2} \rho\left(1-\mathrm{e}^{-k t}\right) / k \\
+\sigma_{2}^{2}\left(1-2 \mathrm{e}^{-k t}+e^{-2 k t}\right) / 2 k^{2} \\
\sigma_{2}^{2}\left(1-\mathrm{e}^{-k t}\right) / k
\end{array}\right)
$$

Therefore, under the risk-neutral measure, $Y_{t}=\ln \left(S_{t}\right)$ is also Gaussian with mean:

$$
\begin{aligned}
Y_{0} & -\delta_{0}\left(1-\mathrm{e}^{-k t}\right) / k+\left(r-\sigma_{Y}^{2} / 2-\alpha^{*}\right) t \\
& +\alpha^{*} k\left(1-\mathrm{e}^{-k t}\right) / k^{2}
\end{aligned}
$$


where $\alpha^{*}=\alpha-\lambda / \kappa$, and variance:

$$
\begin{aligned}
\left(\sigma_{1}^{2}\right. & \left.+\sigma_{2}^{2} / k^{2}-2 \sigma_{1} \sigma_{2} \rho / k\right) t+\left(1-\mathrm{e}^{-2 \kappa t}\right) \sigma_{2}^{2} / 2 \kappa^{3} \\
& +2\left(\rho \sigma_{1} \sigma_{2}-\sigma_{2}^{2} / k\right)\left(1-\mathrm{e}^{-\kappa t}\right) / \kappa^{2}
\end{aligned}
$$

Finally, the spot price, $S_{t}$, is lognormally distributed and therefore the futures price can be written as:

$$
\begin{aligned}
F_{0, T}= & E^{*}\left[S_{T}\right]=\exp \left(E^{*}\left[Y_{T}\right]+\frac{1}{2} \operatorname{Var}^{*}\left[Y_{T}\right]\right) \\
= & \exp \left\{Y_{0}-\delta_{0}\left(1-\mathrm{e}^{-k t}\right) / k\right. \\
& +\left(r-\alpha^{*}+\sigma_{2}^{2} / 2 k^{2}-\sigma_{1} \sigma_{2} \rho / k\right) T \\
& +\left(1-\mathrm{e}^{-2 \kappa t}\right) \sigma_{2}^{2} / 4 \kappa^{3} \\
& \left.+\left(\alpha^{*} k+\rho \sigma_{1} \sigma_{2}-\sigma_{2}^{2} / k\right)\left(1-\mathrm{e}^{-\kappa t}\right) / \kappa^{2}\right\}
\end{aligned}
$$

which is the result already obtained in Schwartz, ${ }^{4}$ equation 20 , but avoiding unnecessary partial differential equations.

Specifically, Schwartz ${ }^{4}$ obtains this result by solving the partial differential equation that must be satisfied by the futures price:

$$
\begin{gathered}
\frac{1}{2} \sigma_{1}^{2} S^{2} F_{S S}+\sigma_{1} \sigma_{2} \rho S F_{S \delta}+\frac{1}{2} \sigma_{2}^{2} F_{\delta \delta}+(r-\delta) S F_{S} \\
+(\kappa(\alpha-\delta)-\lambda) F_{\delta}-F_{T}=0
\end{gathered}
$$

With the terminal boundary condition: $F\left(S_{0}, \delta_{0}, 0\right)$ $=S_{0}$.

This partial differential equation can be obtained in a similar way as in the Black-Scholes
It is clear that the procedure presented here is easier and more straightforward than the one used by Schwartz. ${ }^{4}$

Moreover, using the result in the appendix, the squared volatility of futures returns is:

$$
\begin{aligned}
& \left(\begin{array}{ll}
1 & 0
\end{array}\right)\left(\begin{array}{cc}
1 & \left(\mathrm{e}^{-\kappa T}-1\right) / \kappa \\
0 & \mathrm{e}^{-\kappa T}
\end{array}\right) \\
& \left(\begin{array}{cc}
\sigma_{1}^{2} & \rho \sigma_{1} \sigma_{2} \\
\rho \sigma_{1} \sigma_{2} & \sigma_{2}^{2}
\end{array}\right)\left(\begin{array}{cc}
1 & 0 \\
\left(\mathrm{e}^{-\kappa T}-1\right) / \kappa & \mathrm{e}^{-\kappa T}
\end{array}\right)\left(\begin{array}{l}
1 \\
0
\end{array}\right) \\
& =\sigma_{1}^{2}+\left(1-\mathrm{e}^{-\kappa T}\right)^{2} \sigma_{2}^{2} / \kappa^{2} \\
& \quad-2\left(1-\mathrm{e}^{-\kappa T}\right) \rho \sigma_{1} \sigma_{2} / \kappa
\end{aligned}
$$

which is exactly the formula in Schwartz, ${ }^{4}$ equation 40.

Following the appendix, the discrete-time version of this model can be expressed as:

where

$$
X_{t}=c_{t}+M_{t} X_{t-1}+\psi_{t}
$$

$$
\begin{gathered}
c_{t}=\left(\frac{\left(\mu-\sigma_{1}^{2} / 2-\alpha\right) \Delta t+\alpha\left(1-\mathrm{e}^{-k \Delta t}\right) \lambda / k}{\alpha\left(1-\mathrm{e}^{-k \Delta t}\right)}\right) \\
M_{t}=\left(\begin{array}{cc}
1 & \left(\mathrm{e}^{-\kappa \Delta t}-1\right) / \kappa \\
0 & \mathrm{e}^{-\kappa \Delta t}
\end{array}\right)
\end{gathered}
$$

and $\psi_{t}$ is an $n$-vector of serially uncorrelated Gaussian disturbances with zero mean and variance:

$$
\begin{aligned}
& \operatorname{Var}\left[\psi_{t}\right]= \\
& \left(\begin{array}{cc}
\sigma_{1} \Delta t+\frac{2 \sigma_{1} \sigma_{2} \rho\left(1-\mathrm{e}^{-k \Delta t}-k \Delta t\right)}{k^{2}}-\frac{\sigma_{2}^{2}\left(3-4 \mathrm{e}^{-k \Delta t}+\mathrm{e}^{-2 k \Delta t}-2 k \Delta t\right)}{2 k^{3}} & \frac{\sigma_{1} \sigma_{2} \rho\left(1-\mathrm{e}^{-k \Delta t}\right)}{k}+\frac{\sigma_{2}^{2}\left(1-2 \mathrm{e}^{-k \Delta t}+\mathrm{e}^{-2 k \Delta t}\right)}{2 k^{2}} \\
\frac{\sigma_{1} \sigma_{2} \rho\left(1-\mathrm{e}^{-k \Delta t}\right)}{k}+\frac{\sigma_{2}^{2}\left(1-2 \mathrm{e}^{-k \Delta t}+\mathrm{e}^{-2 k \Delta t}\right)}{2 k^{2}} & \frac{\sigma_{2}^{2}\left(1-\mathrm{e}^{-k \Delta t}\right)}{k}
\end{array}\right)
\end{aligned}
$$

context, that is, we must form an instantaneously riskless portfolio, whose return, in the absence of arbitrage opportunities, must be the risk-free interest rate.
In Schwartz ${ }^{4}$ a discrete-time version of the model is used, which is not the precise one presented above. Specifically, Schwartz ${ }^{4}$ uses a linear approximation to the precise expressions 
presented in this paper. It is easy to show with a Taylor expansion that when $\Delta t \rightarrow 0$ equations (1-3) converge to the linear approximations by Schwartz ${ }^{4}$ (equations 35 in Schwartz ${ }^{4}$ ):

$$
\begin{aligned}
c_{t} & =\left(\begin{array}{c}
\left(\mu-\sigma_{1}^{2} / 2\right) \Delta t \\
\alpha k \Delta t
\end{array}\right), \\
M_{t} & =\left(\begin{array}{cc}
1 & -\Delta t \\
0 & 1-\kappa \Delta t
\end{array}\right) \text { and } \\
\operatorname{Var}\left[\psi_{t}\right] & =\left(\begin{array}{cc}
\sigma_{1}^{2} \Delta t & \sigma_{1} \sigma_{2} \rho \Delta t \\
\sigma_{1} \sigma_{2} \rho \Delta t & \sigma_{2}^{2} \Delta t
\end{array}\right)
\end{aligned}
$$

Therefore, Schwartz ${ }^{4}$ uses a discrete-time version of the model, which is an approximation to the precise one presented above. As we will see below, this accurate discrete-time version of the model, given by expressions (1-3), will have consequences in terms of commodity contingent valuation.

\section{EMPIRICAL IMPLICATIONS}

In order to appreciate the differences between the precise version of the estimates given in this paper and the approximate version in Schwartz, ${ }^{4}$ we have estimated both versions using weekly observations of prices for NYMEX WTI crude oil futures contracts with 1, 3, 5, 7 and 9 months to maturity. Futures from 1/1/1985 to 13/02/ 1995 have been used with a total of 529 observations. This is one of the data sets used in Schwartz. ${ }^{4}$ In that paper, however, the data set includes 510 observations, instead of 529. This is the reason why the results presented here for the Schwartz approximation are not exactly the same as the ones presented in Schwartz. ${ }^{4}$

Table 1 contains some descriptive statistics of the data.

WTI futures prices with one month to maturity are depicted in Figure 1.
Table 1: Data (NYMEX WTI crude oil futures contracts, From 1/1/1985 to 13/02/1995, Weekly Observations)

\begin{tabular}{lll}
\hline $\begin{array}{l}\text { Futures } \\
\text { contract }\end{array}$ & $\begin{array}{l}\text { Mean } \\
(\$ / b b l)\end{array}$ & $\begin{array}{l}\text { Standard } \\
\text { deviation }(\$ / b b l)\end{array}$ \\
\hline F1 & 19.96 & 4.51 \\
F3 & 19.63 & 4.07 \\
F5 & 19.43 & 3.74 \\
F7 & 19.29 & 3.51 \\
F9 & 19.20 & 3.35 \\
\hline
\end{tabular}

Table 2 presents both estimates of the parameters of the two-factor model by Schwartz. ${ }^{4}$ As in Schwartz, ${ }^{4}$ the parameters have been estimated through the Kalman filter methodology. Looking at the table, we can appreciate that the greatest differences are found in the values of the mean-reverting parameter, $\kappa$, in the convenience yield volatility, $\sigma_{2}$, and in the market price of risk associated with the convenience yield, $\lambda$. In fact, we find a value for $\kappa$ considerably lower than the value found with the Schwartz approximation (1.5433 and 1.8855, respectively). Moreover, the value of $\lambda$ found with the precise version is also lower than the value found with the Schwartz approximation (0.2181 and 0.2558, respectively). Finally, the value of $\sigma_{2}$ with the precise and approximate versions is 0.3967 and 0.4622 , respectively. In general, it is found that the approximate version by $\mathrm{Schwartz}^{4}$ tends to overestimate the parameters, since all the values found with the approximate version are higher than the values found with the precise version.

To the best of our knowledge, there is no reliable index that reflects the WTI crude oil spot price. The best approximation for it is the price of NYMEX WTI crude oil futures 


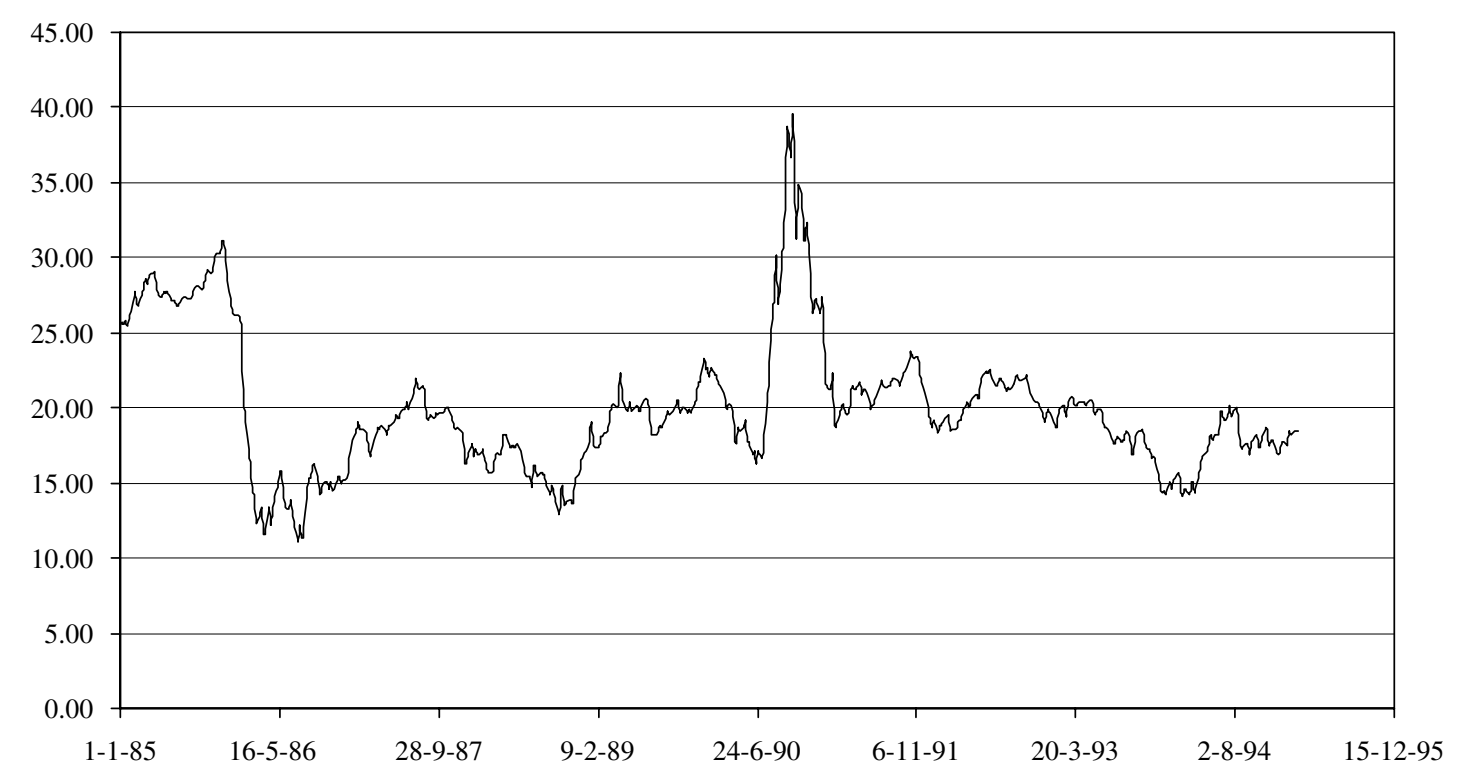

Figure 1: One-month WTI futures price

Table 2: Precise and approximate estimates in the two-factor model by Schwartz ${ }^{4}$

\begin{tabular}{lll}
\hline & Precise version & Schwartz approximation \\
\hline$\mu$ & $0.1629(0.0725)$ & $0.1678(0.0732)$ \\
$k$ & $1.5433(0.0318)$ & $1.8855(0.0356)$ \\
$\alpha$ & $0.1458(0.0558)$ & $0.1496(0.0545)$ \\
$\sigma_{1}$ & $0.3278(0.0073)$ & $0.3293(0.0072)$ \\
$\sigma_{2}$ & $0.3967(0.0113)$ & $0.4622(0.0119)$ \\
$\rho$ & $0.8073(0.0104)$ & $0.8084(0.0107)$ \\
$\lambda$ & $0.2181(0.0864)$ & $0.2558(0.1029)$ \\
\hline
\end{tabular}

Standard errors in parentheses.

contracts with one month to maturity. Table 3 presents the differences between one-month WTI futures prices and the spot price calculated with both the precise and the approximated estimates. The predictive ability of these estimates is compared through two metrics: the mean error (defined as the average of the series of one-month futures prices minus estimated spot prices) and the root mean squared error (RMSE).

It is found that in the full sample period, the precise estimates outperform the approximation by Schwartz, ${ }^{4}$ using the two metrics. They are also the best-performing estimates in all the annual periods considered in the table.

Interestingly, the best performance of the precise estimates is found in 1985 and 1990, years that are characterised by higher volatility (see Figure 1). This is not surprising since one of the main advantages of the precise methodology is that it provides a more accurate estimation of the variance of the residual, $\operatorname{Var}\left[\psi_{t}\right]$, given by expression (3). Moreover, both estimates tend to overestimate spot prices since the mean error is negative in the whole sample period. It is also the case in all the annual periods, except for 1993 and 1994.

Table 4 shows the differences between one-month WTI futures prices and spot prices calculated with both the precise and the 
Table 3: Differences between real and estimated spot price by year

\begin{tabular}{|c|c|c|c|c|}
\hline & \multicolumn{2}{|l|}{ Mean error } & \multicolumn{2}{|c|}{ Root mean squared error } \\
\hline & Precise & Schwartz approximation & Precise & Schwartz approximation \\
\hline 1985-1995 & -0.1559 & -0.1718 & 0.4424 & 0.5031 \\
\hline 1985 & -0.3456 & -0.4158 & 0.4926 & 0.5694 \\
\hline 1986 & 0.0148 & 0.0221 & 0.2092 & 0.2320 \\
\hline 1987 & -0.0728 & -0.0860 & 0.1098 & 0.1218 \\
\hline 1988 & -0.0010 & 0.0059 & 0.1295 & 0.1372 \\
\hline 1989 & -0.2936 & -0.3533 & 0.3249 & 0.3897 \\
\hline 1990 & -0.7200 & -0.7866 & 1.1552 & 1.3055 \\
\hline 1991 & -0.2336 & -0.2610 & 0.3188 & 0.3652 \\
\hline 1992 & -0.1290 & -0.1195 & 0.1691 & 0.1719 \\
\hline 1993 & 0.1376 & 0.1836 & 0.1889 & 0.2322 \\
\hline 1994 & 0.0816 & 0.0906 & 0.1969 & 0.2226 \\
\hline 1995 & -0.0280 & -0.0356 & 0.0898 & 0.1009 \\
\hline
\end{tabular}

The table reports the differences between the one-month futures price and the spot price calculated with precise and approximated estimates, by year.

approximated estimates, by month. As before, the precise estimates outperform the approximation by Schwartz ${ }^{4}$ using the two metrics in all months, except for March with the mean error measure.

Figure 2 compares the improvement in the RMSE, defined as the RMSE computed with the Schwartz approximation minus the RMSE computed with the precise version of the estimates (expressed in percentage), and the standard deviation of one-month futures prices, by month. It is interesting to observe that the months characterised by the highest degree of variance (October and November) show the highest improvement in the RMSE. Again, this is related to the fact that one of the main differences between both methodologies lies on a more accurate estimation of the variance of the residual, $\operatorname{Var}\left[\psi_{t}\right]$, given by expression (3). It should be noted, however, that there are also months with no such high variance showing a high improvement in the RMSE (January and December).

\section{CONCLUSIONS}

In recent years, there have been many papers analysing the stochastic behaviour of commodity prices. Given the complex dynamics of commodity prices, however, the application of the standard Black-Scholes analysis is not straightforward. In fact, these studies present ad hoc solutions, which are very complex and sometimes include approximations.

This paper shows how well-known techniques and results in stochastic calculus can be used to simplify formulae and deductions. Specifically, we have shown how to obtain the 
Table 4: Differences between real and estimated spot prices by month

\begin{tabular}{|c|c|c|c|c|}
\hline & \multicolumn{2}{|l|}{ Mean error } & \multicolumn{2}{|c|}{ Root mean squared error } \\
\hline & Precise & Schwartz approximation & Precise & Schwartz approximation \\
\hline All months & -0.155884852 & -0.171811758 & 0.442441699 & 0.503075855 \\
\hline January & -0.148361764 & -0.176975534 & 0.345867414 & 0.412837467 \\
\hline February & -0.057035493 & -0.063956222 & 0.226770214 & 0.255777129 \\
\hline March & -0.021821794 & -0.018843631 & 0.220816037 & 0.249461653 \\
\hline April & -0.048881713 & -0.057364135 & 0.211696474 & 0.249994592 \\
\hline May & -0.081754769 & -0.085655195 & 0.161309614 & 0.193322674 \\
\hline June & -0.047580096 & -0.050466782 & 0.198748078 & 0.240786749 \\
\hline July & -0.092252959 & -0.095384407 & 0.261123270 & 0.301347232 \\
\hline August & -0.147982930 & -0.153883187 & 0.297399519 & 0.329944708 \\
\hline September & -0.134288566 & -0.142203942 & 0.293639070 & 0.327363269 \\
\hline October & -0.436105927 & -0.482717149 & 0.908492618 & 1.018960284 \\
\hline November & -0.317861875 & -0.347944277 & 0.690954641 & 0.778313141 \\
\hline December & -0.195527578 & -0.222481222 & 0.461966337 & 0.535579146 \\
\hline
\end{tabular}

The table reports the differences between the one-month futures price and the spot price calculated with both precise and approximated estimates, by month.

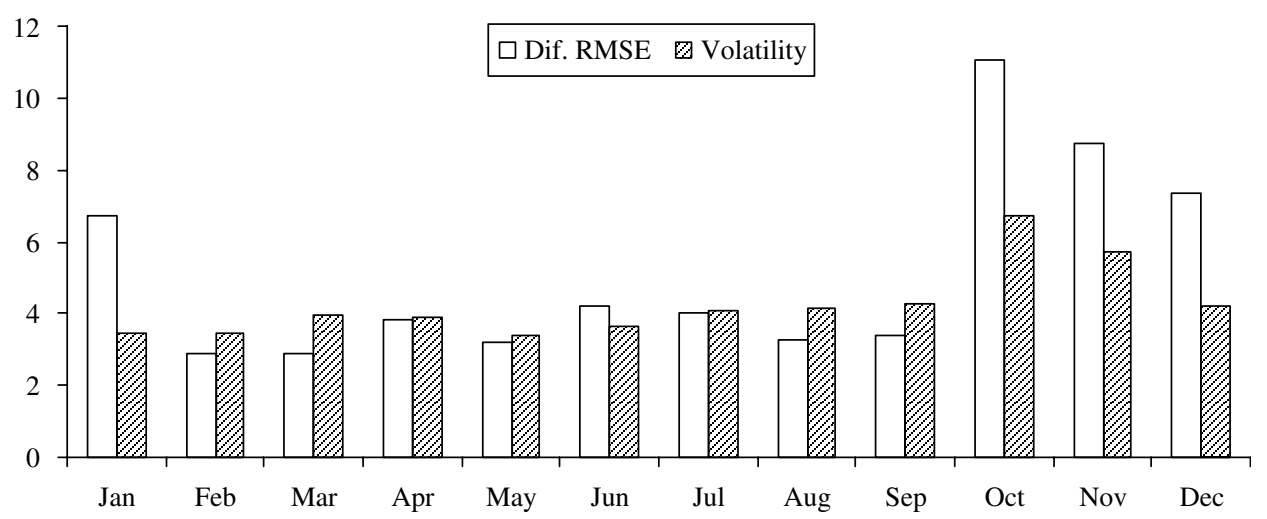

Figure 2: Comparison of the improvement in the RMSE and one-month futures price standard deviation by month

The figure shows the improvement (expressed in percentage) in the RMSE, defined as the RMSE computed with the Schwartz approximation minus the RMSE computed with the precise version of the estimates, and one-month futures price standard deviation, by month. 
expressions in a simpler and accurate way, together with more precise estimates of the parameters than the approximations given by the author in the two-factor model by Schwartz. ${ }^{4}$ It has been shown that the approximations by Schwartz tend to overestimate the parameters and fit the spot prices worse than the precise version. The highest improvements in the RMSE are observed in periods-time characterised by higher volatility. This is related to the fact that one of the main advantages of the precise methodology is that it provides a more accurate estimation of the residual variance in the state-space. These divergences are important in the valuation of commodity contingent claims.

Disclaimer: This paper is the sole responsibility of its authors and the views represented here do not necessarily reflect those of the Banco de España.

\section{Acknowledgments}

We wish to thank Ángel León Valle, Alfonso Novales, Andrés Ubierna Gorricho, Juan Manuel Martín Prieto, Gabriel Paulik and Ana Puente Pérez for their help. We especially thank Repsol YPF for its support. Most of this research was conducted while Javier Población was working in Repsol YPF. We also thank participants in the XXXI Simposio de Análisis Económico in Oviedo. Gregorio Serna acknowledges the financial support provided by the Ministerio de Educación y Ciencia grant SEJ2005-08931C02-01 and Junta de Castilla-La Mancha grant PAI05-074. Any errors that remain are, however, entirely our own.

\section{References}

1 Black, F. and Scholes, M.S. (1972) 'The Valuation of Option Contracts and a Test of Market Efficiency', The Journal of Finance, Vol. 27, pp. 399-418.

2 Brennan, M.J. and Schwartz, E.S. (1985) 'Evaluating Natural Resource Investments', Journal of Business, Vol. 58, pp. 133-155.

3 Paddock, J.L, Siegel, D.R. and Smith, J.L. (1988) 'Option Valuation of Claims on Real Assets: The Case of Offshore Petroleum Leases', Quarterly Journal of Economics, Vol. 103, pp. 479-503.

4 Schwartz, E.S. (1997) 'The Stochastic Behaviour of Commodity Prices: Implication for Valuation and Hedging', The Journal of Finance, Vol. 52, pp. 923-973.

5 Gibson, R. and Schwartz, E.S. (1990) 'Stochastic Convenience Yield and the Pricing of Oil Contingent Claims', The Journal of Finance, Vol. 45, pp. 959-976.

6 Schwartz, E.S. and Smith, J.E. (2000) 'Short-Term Variations and Long-Term Dynamics in Commodity Prices', Management Science, Vol. 46, pp. 893-911.

7 Cortazar, G. and Schwartz, E.S. (2003) 'Implementing a Stochastic Model for Oil Futures Prices', Energy Economics, Vol. 25, pp. 215-238.

8 Cortazar, G. and Naranjo, L. (2006) 'An N-Factor Gaussian Model of Oil Futures Prices', Journal of Futures Markets, Vol. 26, pp. 209-313.

9 Oksendal, B. (1992) 'Stochastic Differential Equations. An Introduction with Applications', 3rd edn. SpringerVerlag, Berlin Heidelberg.

\section{APPENDIX}

\section{Futures Contract Valuation}

Most of the models proposed in the literature assume that the dynamics of a commodity price (or its $\log$ ) is given by a linear stochastic differential system:

$$
\left\{\begin{array}{l}
\mathrm{d} X_{t}=\left(b+A X_{t}\right) \mathrm{d} t+R \mathrm{~d} W_{t} \\
Y_{t}=c X_{t}
\end{array}\right.
$$

where $Y_{t}$ is the commodity price (or its log), $b, A, R$ and $c$ are deterministic parameters independent of $t\left(b \in \mathfrak{R}^{n}, A R \in \mathfrak{R}^{n \times n}, c \in \mathfrak{R}^{n}\right)$ and $W_{t}$ is an $n$-dimensional canonical Brownian motion (ie all components uncorrelated and its variance equal to unity).

The solution of that problem is:

$$
X_{t}=\mathrm{e}^{A t}\left[X_{0}+\int_{0}^{t} \mathrm{e}^{-A s} b \mathrm{~d} s+\int_{0}^{t} \mathrm{e}^{-A s} R \mathrm{~d} W_{s}\right]
$$


It is easy to prove that the solution is unique. 9 Moreover, even in the case that $b, A$ and $R$ were functions of $t$, if $A_{t}$ and $\int_{0}^{t}$. As ds commute, the solution of that problem is (A1).

Accordingly, $X_{t}$ is normally distributed with mean and variance:

$$
\begin{aligned}
E\left[X_{t}\right] & =\mathrm{e}^{A t}\left[X_{0}+\int_{0}^{t} \mathrm{e}^{-A s} b \mathrm{~d} s\right] \\
\operatorname{Var}\left[X_{t}\right] & =\mathrm{e}^{A t}\left[\int_{0}^{t} \mathrm{e}^{-A s} R R^{\prime} \mathrm{e}^{-A s \prime} \mathrm{d} s\right] \mathrm{e}^{A t^{\prime}}
\end{aligned}
$$

With this result, it is easy to prove that the price of a futures contract traded at time $t$ with maturity at time $t+T, F_{t, T}$ can be computed as:

$$
F_{t, T}=\exp \left[c e^{A T} X_{t}+g(T)\right]
$$

where $g$ is a, probably complicated, deterministic function.

\section{Volatility of Futures Returns}

The square volatility of a futures contract traded at time $t$ with maturity at time $t+T$ can be defined as:

$$
\lim _{h \rightarrow 0} \frac{\operatorname{Var}\left[\log F_{t+h, T}-\log F_{t, T}\right]}{h}
$$

Equivalently, it is also possible to define it as

$$
\lim _{h \rightarrow 0} \frac{\operatorname{Var}\left[\log F_{t+h, T-h}-\log F_{t, T}\right]}{h} .
$$

It can be proved that it is the expected value of the square of the coefficient of the Brownian motion $\left(\sigma_{\mathrm{t}}\right)$ in the expansion $\mathrm{d} \log \left(F_{t, T}\right)$

$=\mu_{t} \mathrm{~d} s+\sigma_{t} \mathrm{~d} W_{t}^{F}$, where $W_{t}^{F}$ is a scalar canonical

Brownian motion.

Hence, taking logarithms and differentials on both sides of Equation (A2), it follows that: $\mathrm{d} \log \left(F_{t, T}\right)=c e^{A T} \mathrm{~d} X_{t}=c e^{A T}\left[b+A X_{t}\right] \mathrm{d} t+$ $c e^{A T} R \mathrm{~d} W_{t}$.

Therefore, the squared volatility is:

$$
c e^{A T} R R^{\prime} \mathrm{e}^{A T \prime}{ }^{\prime} c^{\prime}
$$

Note that $R$ need not to be computed as $R R^{\prime}$ is the noise covariance matrix.

\section{Empirical Models}

In the general model presented above, it is easy to prove that knowing $X_{t-1}, X_{t}$ can be written as:

$$
X_{t}=c_{t}+M_{t} X_{t-1}+\psi_{t}
$$

where $c_{t}=e^{A}\left[\int_{t-1}^{t} \mathrm{e}^{-A s} b^{\#} \mathrm{~d} s\right], M_{t}=\mathrm{e}^{A}$ and $\psi_{t}$ is an $n$-vector of serially uncorrelated Gaussian disturbances with zero mean and covariance matrix: $\mathrm{e}^{A}\left[\int_{t-1}^{t} \mathrm{e}^{-A s} R R^{\prime} \mathrm{e}^{-A s^{\prime}} \mathrm{d} s\right] \mathrm{e}^{A^{\prime}}$.

\section{Uses and Remarks}

This general methodology can be used in all kind of problems, is much simpler than the ad hoc solutions presented in the literature, which can only be used in the concrete problem for which they were developed, needing complex procedures like limit steps ${ }^{8}$ or partial differential equations, ${ }^{4}$ and avoids approximations like in Schwartz. ${ }^{4}$ 